\title{
Identify the Key Active Ingredients and Pharmacological Mechanisms of Compound XiongShao Capsule in Treating Diabetic Peripheral Neuropathy by Network Pharmacology Approach
}

\author{
Meixiang Yu, ${ }^{1}$ Xin Song, ${ }^{2}$ Wanhua Yang $\mathbb{D}^{1},{ }^{\text {Ziwei Li, }},{ }^{1}$ Xiaoqin Ma, ${ }^{1}$ and Chenxia Hao ${ }^{1}$ \\ ${ }^{1}$ Department of Pharmacy, Ruijin Hospital, Shanghai Jiaotong University School of Medicine, Shanghai 200025, China \\ ${ }^{2}$ Renji Hospital, Shanghai Jiaotong University School of Medicine, Shanghai 200120, China \\ Correspondence should be addressed to Wanhua Yang; yangwanhuaxy@163.com
}

Received 29 January 2019; Revised 20 March 2019; Accepted 15 April 2019; Published 9 May 2019

Guest Editor: José C. T. Carvalho

Copyright (C) 2019 Meixiang Yu et al. This is an open access article distributed under the Creative Commons Attribution License, which permits unrestricted use, distribution, and reproduction in any medium, provided the original work is properly cited.

Compound XiongShao Capsule (CXSC), a traditional herb mixture, has shown significant clinical efficacy against diabetic peripheral neuropathy (DPN). However, its multicomponent and multitarget features cause difficulty in deciphering its molecular mechanisms. Our study aimed to identify the key active ingredients and potential pharmacological mechanisms of CXSC in treating DPN by network pharmacology and provide scientific evidence of its clinical efficacy. CXSC active ingredients were identified from both the Traditional Chinese Medicine Systems Pharmacology database, with parameters of oral bioavailability $\geq 30 \%$ and drug-likeness $\geq 0.18$, and the Herbal Ingredients' Targets (HIT) database. The targets of those active ingredients were identified using ChemMapper based on 3D-structure similarity and using HIT database. DPN-related genes were acquired from microarray dataset GSE95849 and five widely used databases (TTD, Drugbank, KEGG, DisGeNET, and OMIM). Next, we obtained candidate targets with therapeutic effects against DPN by mapping active ingredient targets and DPN-related genes and identifying the proteins interacting with those candidate targets using STITCH 5.0. We constructed an "active ingredients-candidate targetsproteins" network using Cytoscape 3.61 and identified key active ingredients and key targets in the network. We identified 172 active ingredients in CXSC, 898 targets of the active ingredients, 110 DPN-related genes, and 38 candidate targets with therapeutic effects against DPN. Three key active ingredients, namely, quercetin, kaempferol, and baicalein, and 25 key targets were identified. Next, we input all key targets into ClueGO plugin for KEGG enrichment and molecular function analyses. The AGE-RAGE signaling pathway in diabetic complications and MAP kinase activity were determined as the main KEGG pathway and molecular function involved, respectively. We determined quercetin, kaempferol, and baicalein as the key active ingredients of CXSC and the AGERAGE signaling pathway and MAP kinase activity as the main pharmacological mechanisms of CXSC against DPN, proving the clinical efficacy of CXSC against DPN.

\section{Introduction}

Diabetic peripheral neuropathy (DPN) is one of the most common complications of diabetes, affecting approximately $50 \%$ of people with diabetes [1-3]. Characterized by numbness, pain, paresthesia, and sensory loss, it becomes a major cause of disability and mortality [4-6]. Given the estimated global prevalence of diabetes of 693 million by 2045 [4], DPN is likely to affect as many as 346.5 million people worldwide, at a tremendous cost.
The pathogenesis of DPN is complex and multifactorial $[7,8]$. It is generally accepted that chronic hyperglycemia induces the generation of advanced glycation end products (AGEs) in peripheral nervous tissue, and AGEs frequently result in neurological dysfunction mainly by modifying nervous structural proteins or overexpressing their receptors (RAGE) [9-11]. In addition, high glucose level activates mitogen-activated protein kinase (MAPK) in sensory neurons [8]. Moreover, elevated p38 and ERK activities may 


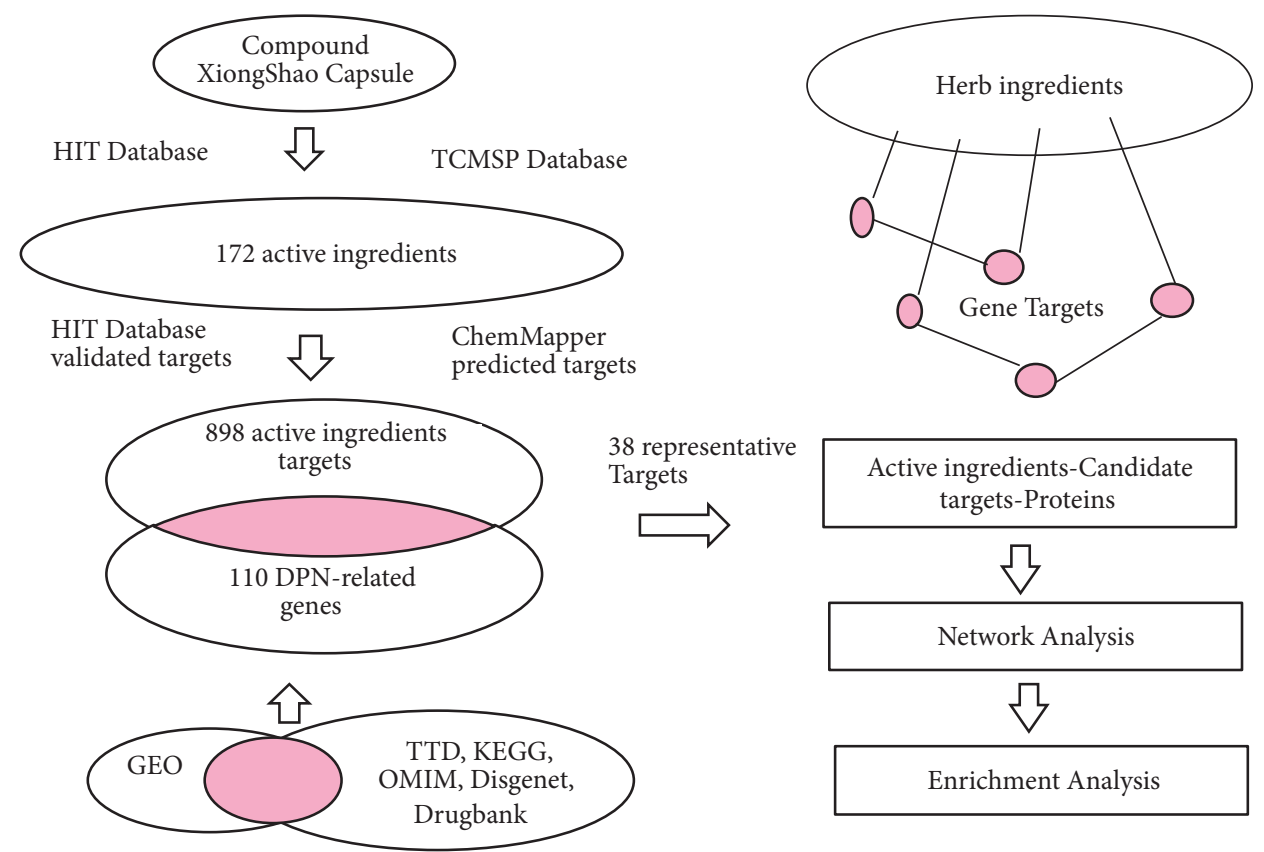

Representative ingredients and targets against DPN

The pharmacological mechanisms of CXSC

FIGURE 1: Flowcharts of the network pharmacology analysis. Left: summary of the identification of representative ingredients of CXSC and targets with therapeutic effects against DPN. Right: summary of the determination of the pharmacological mechanisms of CXSC.

cause neuron apoptosis, affecting neuronal functions and accelerating the progress of DPN $[8,12]$.

Despite advancements in the understanding of DPN pathogenesis, prevention and therapy of DPN mainly focus on glucose control and lifestyle modification [13]. There is still a lack of therapies against DPN pathogenesis other than aldose reductase inhibitors, i.e., epalrestat, and antioxidants, i.e., alpha-lipoic acid $[14,15]$. In addition, these therapies show modest efficacy in clinical practice and exhibit certain adverse reactions, such as nausea, vomiting, and dizziness $[14,16]$. Thus, safe and effective treatments of DPN that act on its mechanisms are urgently needed [13].

Recently, Traditional Chinese Medicine (TCM) has shown efficacy in treating DPN $[17,18]$. Compound XiongShao Capsule (CXSC) was produced from Buyang Huanwu Decoction which has been known for hundreds of years to possess significant neuroprotective properties [19-21] and certain clinical effect against DPN $[22,23]$. CXSC has also been approved to use to treat DPN in Ruijin Hospital, Shanghai Jiao Tong University by the Shanghai Food and Drug Administration. CXSC is composed of 12 traditional Chinese herbs: Radix Paeoniae (RP), Radix Cyathulae (RC), Rhizoma Chuanxiong (RCX), Cortex Lyci (CL), Radix Saposhnikoviae (RS), Cassia Twig (CT), Sargassum pallidum (SP), Polygonatum sibiricum (PG), Astragali Radix (AgR), Ramulus mori (RM), Silybum marianum (SM), and Orostachys fimbriata (OF). CXSC has shown significant efficacy in clinical practice over several years, including improving whole-blood highshear viscosity and improving incubation period and amplitude in the median or peroneal nerve of patients suffering from DPN [24]. Furthermore, some herbs in CXSC have been confirmed to exhibit positive pharmacological effects against DPN. For example, CL, AgR, and SM are involved in blood glucose control and show anti-inflammatory and inhibitory effects against oxidative stress [25-27].

However, the active ingredients of CXSC and their potential pharmacological mechanisms have not been fully studied. In a previous study, network pharmacology was used to discover the active ingredients and elucidate the mechanisms of herbal formulae [28]. Thus, our study aimed to use the network pharmacology approach to identify the key active ingredients of CXSC and their pharmacological mechanisms in treating DPN. A flowchart of the network pharmacology approach is presented in Figure 1.

\section{Materials and Methods}

2.1. Identification of Active Ingredients. Active ingredients of CXSC were collected both from the Traditional Chinese Medicine Systems Pharmacology (TCMSP) Database [29] (http://lsp.nwu.edu.cn/, updated on May 31, 2014) according to the ADME parameters: oral bioavailability $(\mathrm{OB})>30 \%$ and drug-likeness $(\mathrm{DL})>0.18$ [30] and from the Herbal Ingredients' Targets (HIT) Database [31] (http://lifecenter.sgst .cn/hit/, downloaded on July 31, 2018). By combining these two databases and removing any overlapping data, the active ingredients of CXSC were identified.

The TCMSP database is a unique TCM platform for identifying the relationships between drugs, targets, and diseases [29]. HIT is a database containing herbal ingredients and validated protein targets derived from more than 3250 literatures [31]. 
TABLE 1: The number of overlapped active ingredients between herbs.

\begin{tabular}{lcc}
\hline Active ingredients & Total & Herbs \\
\hline beta-sitosterol & 7 & $C T, C L, O F, P G S, R C, R P, R S$ \\
Sitosterol & 6 & $C T, O F, P G S, R P, R S, R C X$ \\
quercetin & 5 & $S M, A g R, O F, R C, S P$ \\
Stigmasterol & 3 & $S M, C L, R P$ \\
acetic acid & 3 & $A g R, C T, R P$ \\
kaempferol & 3 & $A g R, O F, R M$ \\
$(-)$-taxifolin & 2 & $S M, C T$ \\
CLR & 2 & $S M, C L$ \\
hederagenin & 2 & $A g R, C L$ \\
FA & 2 & $A g R, R C X$ \\
(+)-catechin & 2 & $C T, R P$ \\
baicalein & 2 & $P G S, R P$ \\
ecdysterone & 2 & $R C, R M$ \\
Mandanol & 2 & $R S, R C X$ \\
\hline
\end{tabular}

2.2. Targets of Active Ingredients. The validated targets of the active ingredients of CXSC were collected from the HIT database $[32,33]$. In addition, the predicted targets of these active ingredients were obtained by using ChemMapper (http://www.lilab-ecust.cn/chemmapper/index.html), a web server for predicting potential drug targets based on $3 \mathrm{D}$ structure similarity [34], with 3D structure similarity of above 1.0 and prediction score of above $0[32,33]$. Duplicates of the validated and predicted targets were eliminated, and the targets of the active ingredients of CXSC were screened.

2.3. Genes Related to DPN Obtained. Known genes of DPN were identified from five currently available databases using "diabetic peripheral neuropathy" as the keyword. In addition, the main differentially expressed genes (DEGs) between DPN patients and diabetic patients were extracted from microarray data GSE95849 [35] in the Gene Expression Omnibus (GEO; http://www.ncbi.nlm.nih.gov/geo/) database [36], with a cutoff value of $\mathrm{P}<0.05$ and fold change $|\mathrm{FC}|$ of $\geq 1.5$ [37]. DPNrelated genes were identified after removal of duplicates.

The five databases were: the Therapeutic Target Database [38] (TTD, http://bidd.nus.edu.sg/group/cjttd/, last updated: 15th Sep. 2017); DrugBank [39] (http://www.drugbank.ca/, version:5.10); the Kyoto Encyclopedia of Genes and Genomes Pathway Database [40] (KEGG, https://www.kegg.jp/, downloaded:June. 2018); DisGeNET Database [41] (http://www .disgenet.org/web/DisGeNET/menu/home, version:5.0); Online Mendelian Inheritance in Man Database [42] (OMIM, http://www.omim.org/, last updated: 30th June. 2018).

\subsection{Candidate Targets and Proteins Interacting with Candidate} Targets. Candidate targets with therapeutic effects against DPN were identified by mapping the targets of the active ingredients of CXSC and the genes related to DPN [33]. The proteins that interacted with these candidate targets were screened by using STITCH 5.0 (http://stitch.embl.de/) [43], a database of known and predicted interactions between chemicals and proteins, with the species limited to "Homo Sapiens" and a confidence score of $>0.9$.
2.5. Network Construction and Enrichment Analysis. A network of "active ingredients-candidate targets-proteins" was constructed using the Cytoscape 3.61 software [44]. The key active ingredients of CXSC and the key targets in this network were simultaneously identified by Network Analyzer plugin [45] using the following criterion: nodes with degree values exceeding twice the average value of all nodes in the network [46]. The degree value is the number of edges a node has in a network, which indicates how many active ingredients/targets/proteins one active ingredient/target/protein is related with. The larger the degree value, the more critical a role the node (active ingredient, target, or protein) is believed to play in the network [46]. A widely used visualization software, ClueGO [47], was used to explore the potential KEGG pathways and molecular functions of the key targets.

\section{Results}

3.1. Active Ingredients of CXSC. A total of 172 active ingredients (Supplementary Table 1) were identified in this study, of which 133 were collected from the TCMSP database and 39 were obtained from the HIT database. The numbers of active ingredients in RP, AgR, RCX, RS, CL, CT, PGS, SM, $\mathrm{RM}, \mathrm{RC}, \mathrm{OF}$, and SP were $35,22,22,21,21,13,13,13$, $12,7,5,5$, and 4 , respectively. The active ingredients that overlapped between herbs are shown in Table 1; for example, beta-sitosterol was identified in seven herbs, sitosterol was identified in six herbs, and quercetin was identified in five herbs.

3.2. Targets of the Active Ingredients of CXSC. A total of 898 targets of the active ingredients of CXSC (Supplementary Table 2) were identified in our study, including 229 validated targets and 669 predicted targets. Our analysis results showed that quercetin, baicalein, and kaempferol were the top three active ingredients targeting 308, 296, and 224 targets, respectively. Furthermore, there were 140 common targets of 12 herbs, 72 common targets of 11 herbs, 30 common targets of 10 herbs, 26 common targets of 9 herbs, 33 common targets 


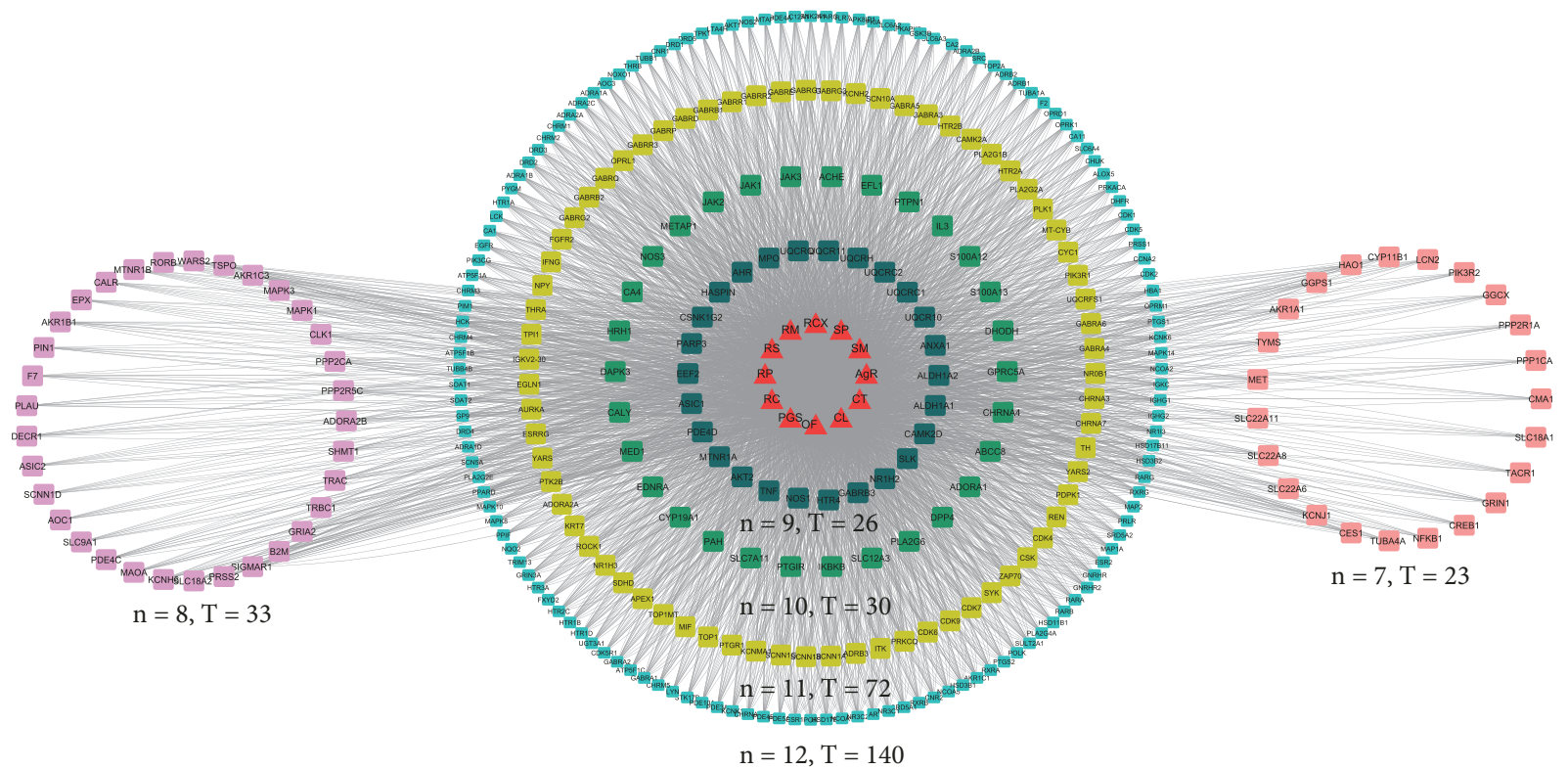

Figure 2: The targets that overlapped between at least 7 herbs. The triangle nodes represent the herbs, whereas the rectangle nodes represent the targets. The targets distributed in a circle are targeted by the same number of herbs. The number of herbs and number of targets are expressed as "n" and "T," respectively.

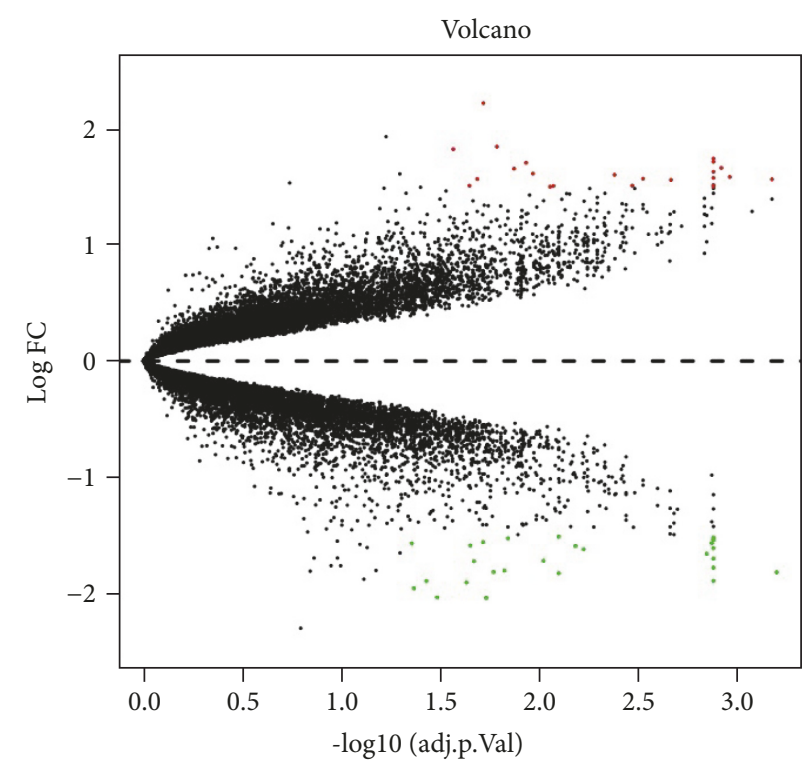

FIGURE 3: Volcano map of 51 DEGs. Red nodes represent upregulated genes and green nodes represent downregulated genes.

of 8 herbs, and 23 common targets of 7 herbs. The common targets of at least 7 herbs are shown in Figure 2.

3.3. Genes Related to DPN. A total of 51 DEGs were extracted from microarray data GSE95849, including 23 upregulated genes and 28 downregulated genes, as shown in Figure 3. After combination with the 59 known targets from the other five databases, a total of 110 DPN-related genes were identified (Supplementary Table 3).

3.4. Candidate Targets and Interacting Proteins. A total of 38 candidate targets were shown to have potential pharmacological effects against DPN, including 11 predicted targets (INS, MAPK14, MMP2, NOS3, SCN9A, SLC6A4, SLC6A3, OPRM1, TUBB1, ABCC8, and KCNJ11) and 27 validated targets (AKT1, BAX, BCL2, CASP3, JUN, MAPK1, MAPK8, TNF, VEGFA, IL1B, IL6, PRKCA, PRKCB, RELA, SELE, STAT1, PLAU, CCND1, COL1A1, CXCL8, EDN1, F3, ICAM1, ACE, SLC6A2, and CYP1A2). Specifically, TNF, IL1B, IL6, and CXCL8 were involved in inflammatory reactions; AKT1, BAX, BCL2, CASP3, and MAPK8 were related to apoptosis; and MAPK1, MAPK8, and MAPK14 were associated with MAPK activity. In addition, there were 86 proteins that interacted with those candidate targets. 


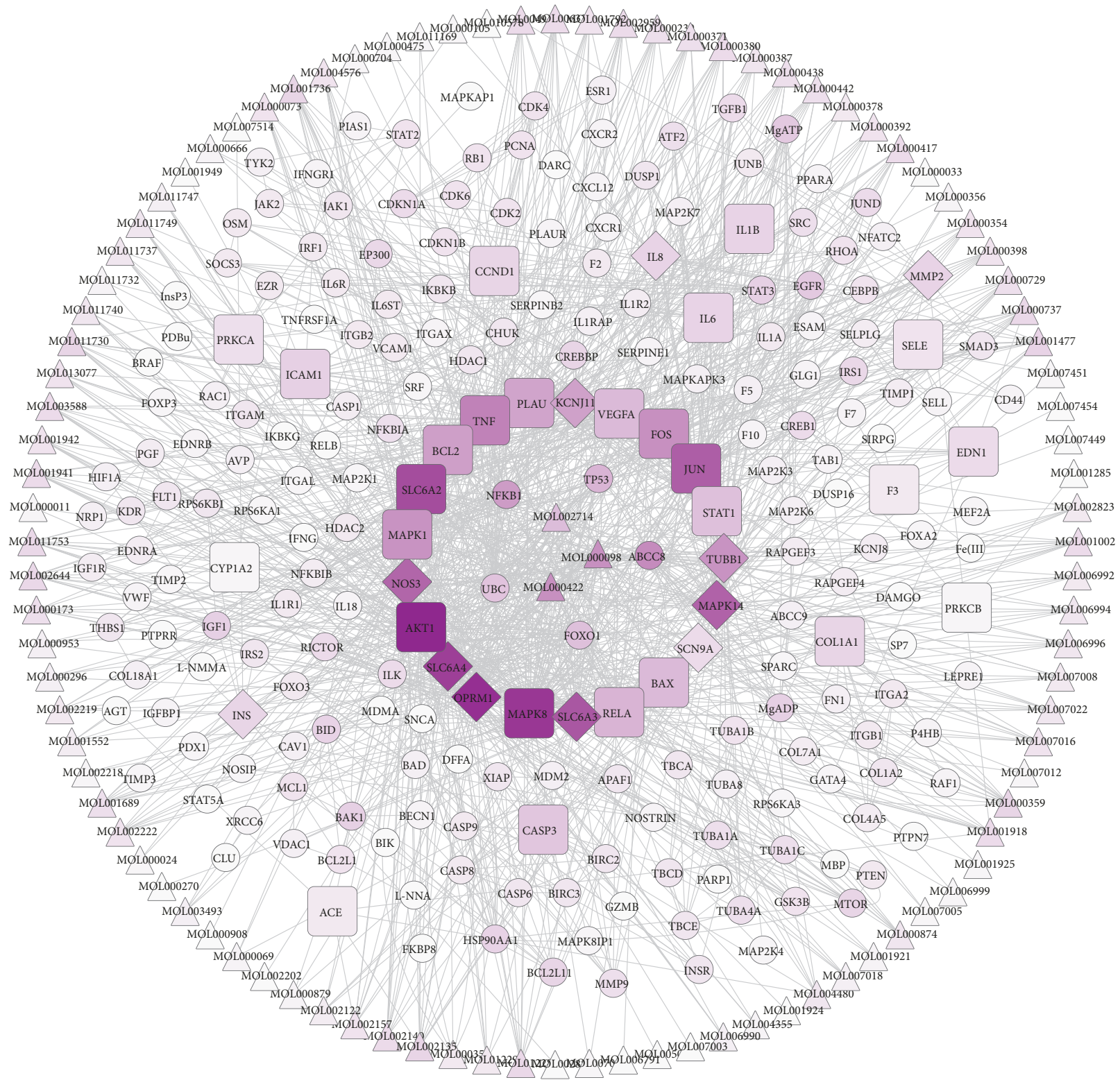

Figure 4: "Active ingredients-candidate targets-proteins" network. The triangle nodes represent active ingredients, the rectangle nodes represent validated targets, the diamond nodes represent predicted targets, and the circular nodes represent interacting proteins. The color of the nodes is shown in a gradient from purple to transparent according to descending order of the degree value. The three key active ingredients and 25 key targets are listed in the center circle.

3.5. Key Active Ingredients and Key Targets in the Network. An "active ingredients-candidate targets-proteins" network of 38 candidate targets and their interacting proteins was constructed using the Cytoscape 3.61 software (Figure 4). There were 328 nodes and 1396 edges in the network, with an average degree value of 8.767 , as calculated by using the NetworkAnalyzer plugin. There were 28 nodes with degree values exceeding 18 (twice the average value of the degree), comprising of three active ingredients (quercetin, kaempferol, and baicalein) representing the key active ingredients (Figure 5) and 25 targets representing the key targets (Table 2).
3.6. KEGG Pathways and Molecular Functions Enriched. The enrichment analysis results showed enrichment in the KEGG signaling pathways and molecular functions. As shown in Figure 6, the following three KEGG pathways were enriched: the AGE-RAGE signaling pathway in diabetic complications, $B$ cell receptor signaling pathway, and cocaine addiction pathway, with the AGE-RAGE signaling pathway accounting for $88 \%$ of all the pathways enriched. The enriched molecular functions were divided into three groups: MAP kinase activity, phosphatase binding, and neurotransmitter: sodium symporter activity (Figure 7). 
<smiles>O=c1c(O)c(-c2ccc(O)c(O)c2)oc2cc(O)cc(O)c12</smiles>

Quercetin (MOL000098)<smiles>O=c1cc(-c2ccccc2)oc2cc(O)c(O)c(O)c12</smiles>

Baicalein (MOL002714)<smiles>O=c1c(O)c(-c2ccc(O)cc2)oc2cc(O)cc(O)c12</smiles>

Kaempferol (MOL000422)

FIGURE 5: The chemical structure of the 3 key active ingredients.

TABLE 2: The information about the 25 key targets.

\begin{tabular}{|c|c|c|c|c|}
\hline Gene & UniProt & Description & Degree & Source \\
\hline AKT1 & P31749 & RAC-alpha serine/threonine-protein kinase & 83 & validated \\
\hline OPRM1 & P35372 & Mu-type opioid receptor & 76 & predicted \\
\hline MAPK8 & P45983 & Mitogen-activated protein kinase 8 & 72 & validated \\
\hline SLC6A4 & P31645 & Sodium-dependent serotonin transporter & 67 & predicted \\
\hline SLC6A2 & P23975 & Sodium-dependent noradrenaline transporter & 60 & validated \\
\hline SLC6A3 & Q01959 & Sodium-dependent dopamine transporter & 56 & predicted \\
\hline JUN & P05412 & Transcription factor AP-1 & 53 & validated \\
\hline MAPK14 & Q16539 & Mitogen-activated protein kinase 14 & 51 & predicted \\
\hline NOS3 & P29474 & Nitric oxide synthase & 50 & predicted \\
\hline TNF & P01375 & Tumor necrosis factor & 40 & validated \\
\hline ABCC8 & Q09428 & ATP-binding cassette sub-family $\mathrm{C}$ member 8 & 37 & proteins \\
\hline FOS & P01100 & Proto-oncogene c-Fos & 35 & validated \\
\hline TUBB1 & Q9H4B7 & Tubulin beta-1 chain & 34 & predicted \\
\hline MAPK1 & P28482 & Mitogen-activated protein kinase 1 & 34 & validated \\
\hline NFKB1 & P19838 & Nuclear factor NF-kappa-B p105 subunit & 31 & proteins \\
\hline BCL2 & P10415 & Apoptosis regulator Bcl-2 & 30 & validated \\
\hline KCNJ11 & Q14654 & ATP-sensitive inward rectifier potassium channel 11 & 29 & predicted \\
\hline PLAU & P00749 & Urokinase-type plasminogen activator & 28 & validated \\
\hline RELA & Q04206 & Transcription factor $\mathrm{p} 65$ & 23 & validated \\
\hline TP53 & P04637 & Cellular tumor antigen p53 & 23 & proteins \\
\hline BAX & Q07812 & Apoptosis regulator BAX & 21 & validated \\
\hline VEGFA & P15692 & Vascular endothelial growth factor A & 21 & validated \\
\hline STAT1 & P42224 & Signal transducer and activator of transcription 1-alpha/beta & 19 & validated \\
\hline FOXO1 & Q12778 & Forkhead box protein $\mathrm{O} 1$ & 19 & proteins \\
\hline UBC & P0CG48 & Polyubiquitin-C & 18 & proteins \\
\hline
\end{tabular}

\section{Discussion}

TCM pharmacological mechanisms have always been associated with multiple components and multiple targets that are difficult to explain. However, the holistic ideas of TCM have been revealed by emerging network pharmacology approaches studying the relationships between drugs, targets, and diseases [28]. In our study, the active ingredients of CXSC, targets of these active ingredients, and genes related to DPN were identified comprehensively from the databases. Next, an "active ingredient-target-protein" network with 328 nodes and 1396 edges was constructed by the Cytoscape software. After analyzing the network, three key active ingredients and 25 key targets of CXSC were determined, and the pharmacological mechanisms of CXSC in treating DPN were elucidated by enrichment analysis of the $25 \mathrm{key}$ targets.

4.1. Quercetin, Kaempferol, and Baicalein as the Key Active Ingredients of CXSC. The "active ingredients-targets-proteins" network showed quercetin, kaempferol, and baicalein as the key active ingredients of CXSC that exhibit therapeutic effects against DPN. Moreover, these key ingredients targeted 308 (34.3\%), 296 (33.0\%), and $244(27.2 \%)$ targets, respectively. Furthermore, these three key ingredients targeted 22 of the 38 candidate targets with therapeutic effects against 

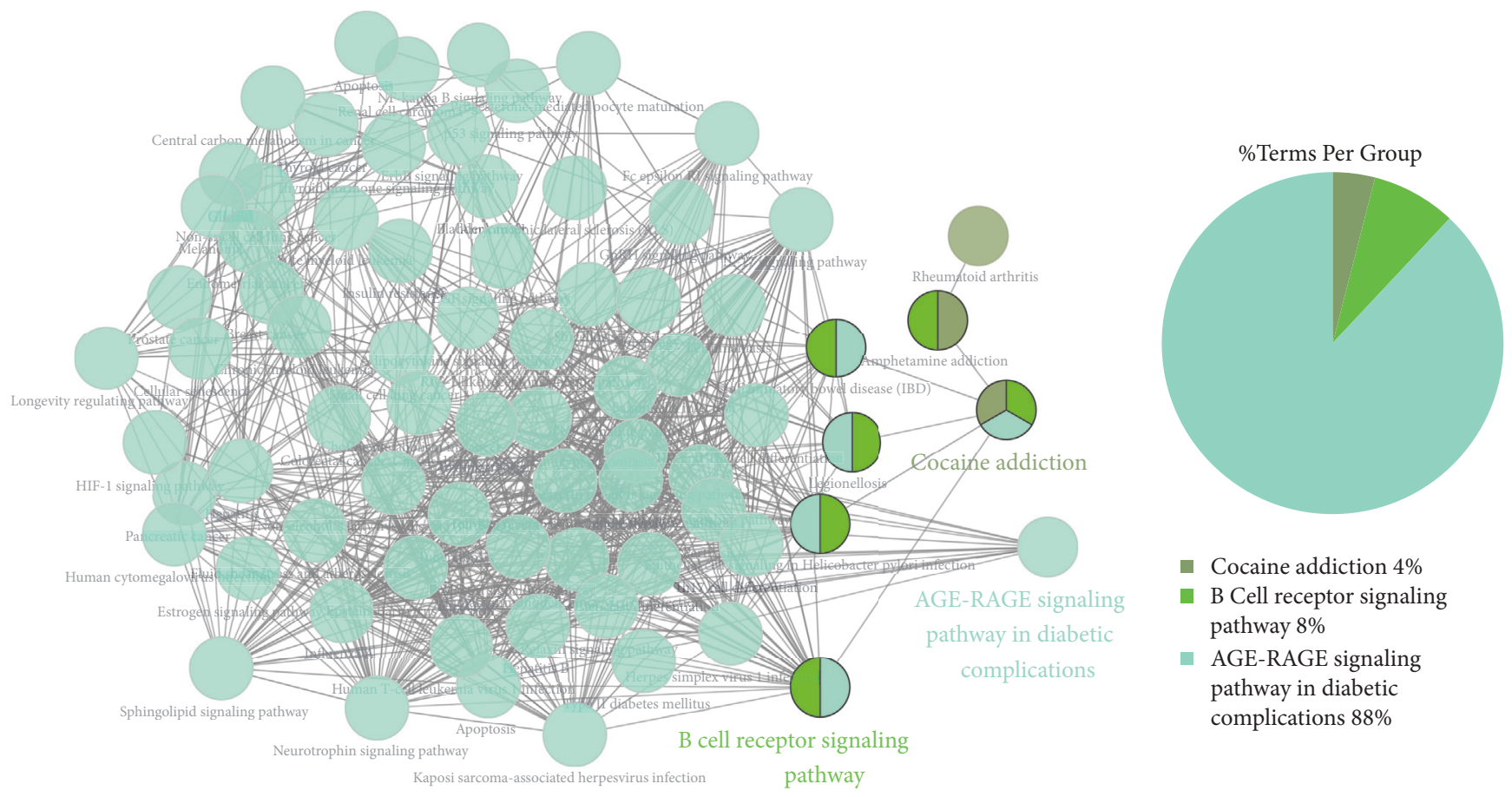

FIGURE 6: KEGG signaling pathways.
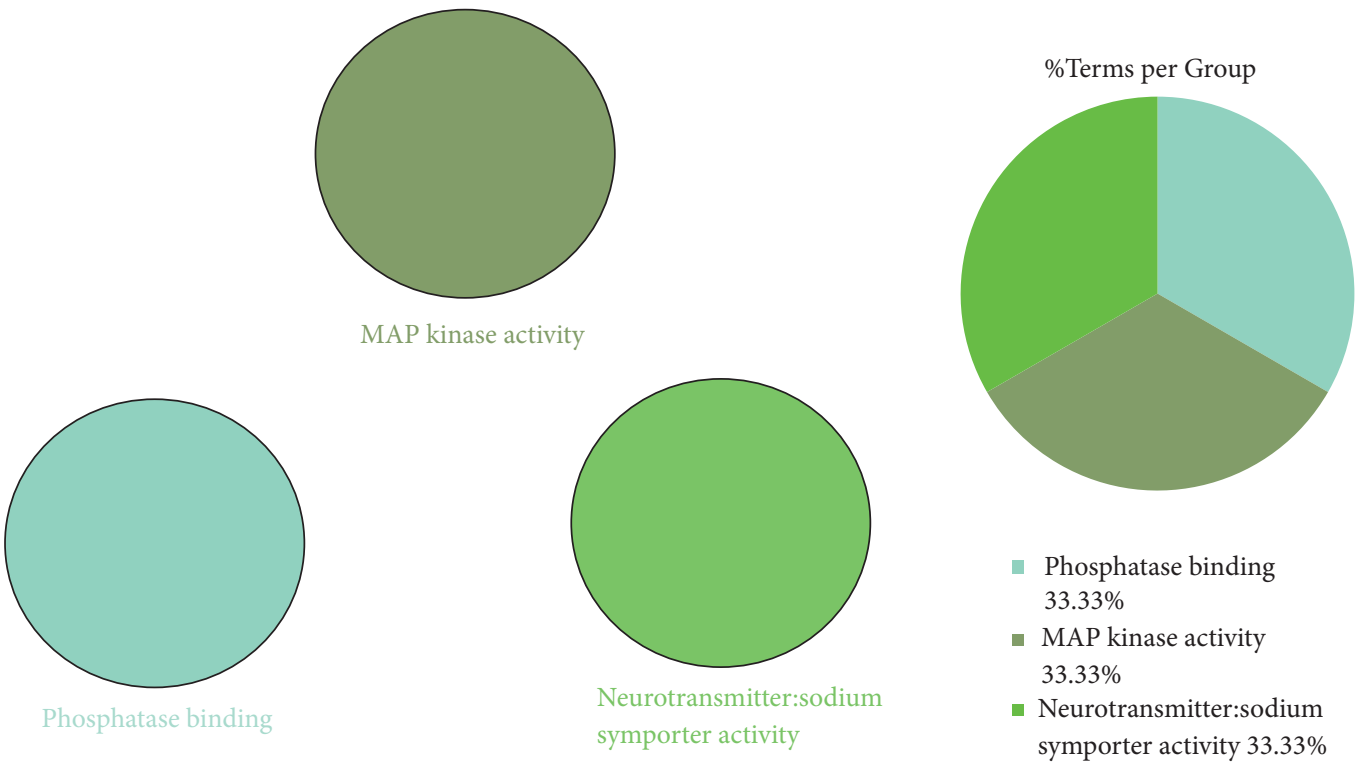

Figure 7: Molecular functions analysis.

DPN, as well as 21 of the 25 key targets of CXSC, as shown in Figure 8. Interestingly, several studies have previously shown that quercetin may be helpful against diabetic neuropathy by attenuating cold allodynia and hyperalgesia [48, 49], maintaining the density of the general neuronal population [50], and restoring sciatic nerves injuries [51] in streptozotocin(STZ-) induced diabetic rats. Kaempferol [52] and baicalein [53] also showed neuroprotective effect against STZ-induced diabetic neuropathy. Thus, we speculated that CXSC exhibited significant therapeutic effect against DPN mainly through the synergistic actions of quercetin, kaempferol, and baicalein.

4.2. Regulations of the AGE-RAGE Pathway and MAPK Activity as the Pharmacological Mechanisms of CXSC Therapeutic Effect against DPN. Kaempferol and baicalein, the key active ingredients of CXSC, were previously confirmed to reduce the formation of AGEs, thereby reducing inflammatory responses in diabetic rat nerves $[52,54]$; thus, these two compounds may exert therapeutic effect against DPN. 


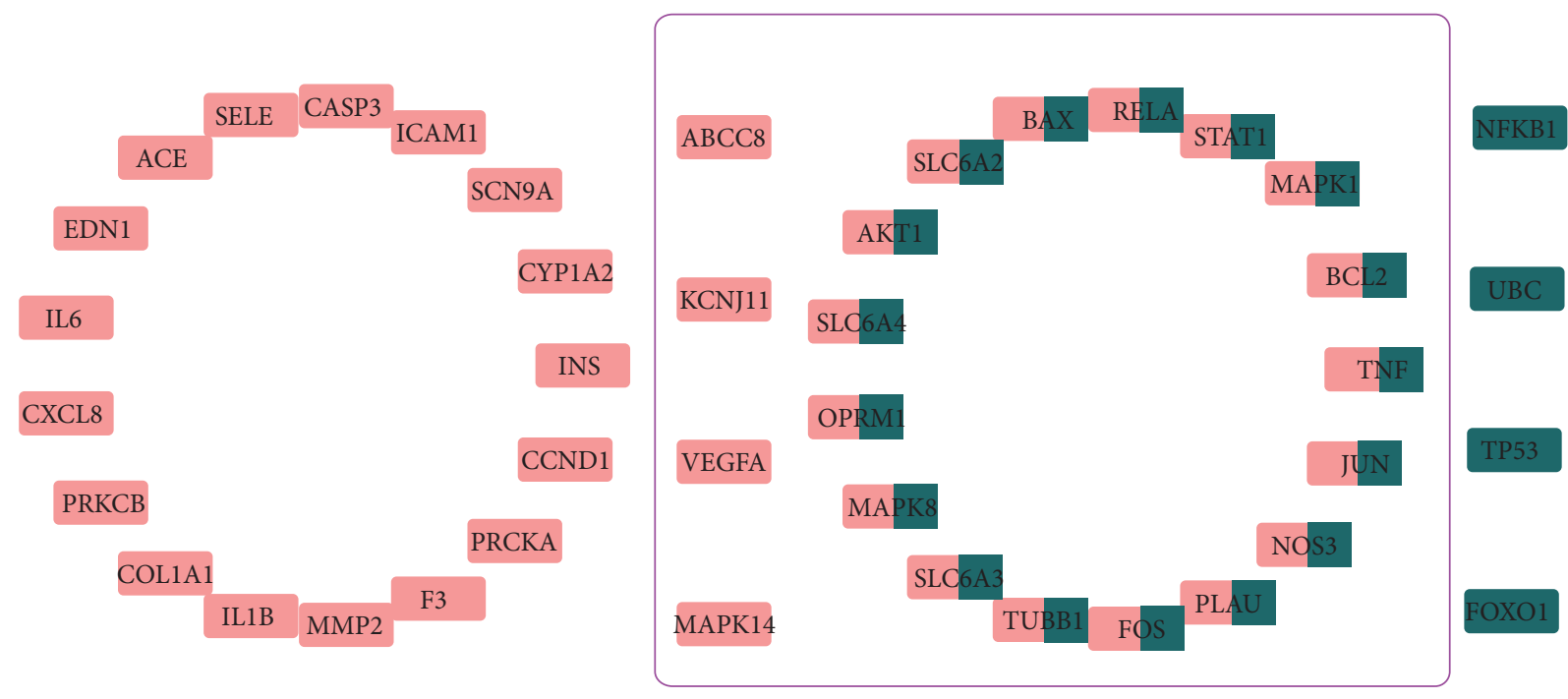

FIGURE 8: Data of the three key active ingredients (quercetin, kaempferol, and baicalein). Pink rectangles represent 38 candidate targets with therapeutic effects against DPN, whereas green rectangles represent the 25 key targets. Two-colored rectangles are targets that overlapped between the two categories. Targets in the purple box are targets of quercetin, kaempferol, and baicalein.

Quercetin, another key active ingredient of CXSC, protected rat dorsal root ganglion neurons against high glucoseinduced injury in vitro through dose-dependent inhibition of the NF- $\kappa \mathrm{B}$ signaling pathway; thus, this compound may be beneficial as a treatment of diabetic neuropathy [55]. Furthermore, activation and perpetuation of the AGE-RAGE signaling pathway have been reported in diabetic neuropathy [9]. AGEs act on RAGEs to allow sustained activation of $\mathrm{NF}-\kappa \mathrm{B}$, which aggravates inflammatory reactions and leads to neuronal dysfunction [9, 11]. Lukic I.K [10] identified the AGE-RAGE signaling pathway as a new therapeutic target of neuronal dysfunction. In our study, the AGERAGE signaling pathway of diabetic complications accounted for $88 \%$ of all the enriched pathways, suggesting that the therapeutic effect of CXSC against DPN was mainly related to reduction in AGE formation and inhibition of $\mathrm{NF}-\kappa \mathrm{B}$.

In addition, AGEs can activate p38 MAPK, leading to neuron apoptosis [56]. MAPK may play important roles in neuronal cell death or regeneration $[57,58]$. For example, p38 MAPK was reported to aggravate oxidative stress and inflammation through induction of nitric oxide synthase, thereby resulting in $\mathrm{NO}$ production, as well as through regulation of the production of cytokines, such as TNF and IL-10, leading to neuronal cell death $[59,60]$. Notably, increased phosphorylation of MAPK has been detected in diabetic animal models [61-63] and in the peripheral nerves of patients with DPN [12]. p38 MAPK, especially has been proved to be associated with DPN pathogenesis, such as increased mechanical hyperalgesia [64-66] and reduced nerve conduction velocity [67]. Thus, adjustment in the activity of MAPK may be the basis of preventive treatments of diabetic neuropathy [9]. Fortunately, tetramethylpyrazine, a main ingredient of RCX in CXSC, was found to block MAPK and suppress reactive oxygen species in N9 microglial cells, which may shed light on future treatments of neurodegenerative diseases [68].
Baicalein, one of the three key active ingredients identified in the current study, counteracts diabetes-associated p38 MAPK phosphorylation and oxidative-nitrosative stress, targeting several mechanisms implicated in DPN [53]. Therefore, the effects of CXSC on MAPK regulation against DPN may be attributed to tetramethylpyrazine and baicalein. Our molecular function analysis also indicated that the therapeutic effect of CXSC against DPN was related to MAPK activity. Our results were consistent with those of previous studies, which indicated that p38 MAPK activation mediates RAGEinduced, NF- $\kappa \mathrm{B}$-dependent secretion of proinflammatory cytokines, leading to accelerated inflammation. Activation of the AGE-RAGE pathway and MAPK appeared to be present not only in DPN but also in diabetic nephropathy [69] and diabetic keratopathy [56]. Thus, further studies are needed to investigate whether CXSC is effective against other diabetic complications.

\section{Conclusions}

In conclusion, the multicomponent and multitarget features of the therapeutic effects of CXSC against DPN were effectively elucidated through network pharmacology approach. Quercetin, kaempferol, and baicalein were determined as the key active ingredients of CXSC. In addition, the AGERAGE signaling pathway and regulation of MAPK activity were shown as the main pharmacological mechanisms of the therapeutic effects of CXSC against DPN, thereby providing scientific evidence of the clinical efficacy of CXSC against DPN.

\section{Data Availability}

The data of our research can be acquired from the Supplementary Materials uploaded with this article. 


\section{Conflicts of Interest}

The authors declare no conflicts of interest.

\section{Authors' Contributions}

Yu Meixiang and Yang Wanhua designed, wrote, and revised the manuscript. Yu Meixiang and Song Xin performed the experiments; Li Ziwei, Ma Xiaoqin, and Hao Chenxia revised the manuscript and provided technical or material support.

\section{Acknowledgments}

This project was supported by the key scientific research projects of the Science and Technology Commission of Shanghai (no.17401901100).

\section{Supplementary Materials}

Supplementary Table 1. 172 active ingredients in Compound XiongShao Capsule. 172 active ingredients in Compound XiongShao Capsule were downloaded from both the Traditional Chinese Medicine Systems Pharmacology (TCMSP) Database (http://lsp.nwu.edu.cn/, updated on May 31, 2014) according to an ADME principle: oral bioavailability $(\mathrm{OB})>$ $30 \%$ and drug-likeness (DL) $>0.18$ and the Herbal Ingredients' Targets (HIT) Database (downloaded on July 31, 2018). Supplementary Table 2. 898 targets of active ingredients in Compound XiongShao Capsule. The validated targets of the active ingredients in Compound XiongShao Capsule were extracted from HIT database. At the same time, the predicted targets of the active ingredients, which were saved as "MOL2" file format and then inputted into the ChemMapper (http://www.lilab-ecust.cn/chemmapper/index.html, downloaded in August 2018), were selected if the 3D similarity was above 1.0 and the prediction score was $>0$ as well. Eliminate duplicates, respectively, the targets of active ingredients were obtained. Supplementary Table 3. 110 diabetic peripheral neuropathy-related genes. The main differentially expressed genes (DEGs) of diabetic peripheral neuropathy (DPN) were extracted from Microarray data GSE95849 including six diabetic samples and six DPN samples in the Gene Expression Omnibus database (GEO, http://www.ncbi.nlm.nih.gov/geo/) with cut-off values of $\mathrm{P}$ $<0.05$ and fold change $|F C| \geq 1$.5. Simultaneously, known genes related to DPN were obtained from five currently available databases using "diabetic peripheral neuropathy" as the keyword: (1) the Therapeutic Target Database (TTD) (http://bidd.nus.edu.sg/group/cjttd/, last updated:15th Sep. 2017). (2) DrugBank (http://www.drugbank.ca/, version: 5.10). (3) the Kyoto Encyclopedia of Genes and Genomes (KEGG) Pathway Database (https://www.kegg.jp/, downloaded:June. 2018) (4) DisGeNET Database (http://www .disgenet.org/web/DisGeNET/menu/home, version:5.0). (5) Online Mendelian Inheritance in Man (OMIM) Database (http://www.omim.org/, last updated:30th June. 2018). At last, DPN-related genes were collected after eliminating duplicates. (Supplementary Materials)

\section{References}

[1] M. Turns, "The diabetic foot: An overview for community nurses," British Journal of Community Nursing, vol. 17, no. 9, pp. 422-433, 2012.

[2] C. J. M. Alleman, K. Y. Westerhout, M. Hensen et al., "Humanistic and economic burden of painful diabetic peripheral neuropathy in Europe: a review of the literature," Diabetes Research and Clinical Practice, vol. 109, no. 2, pp. 215-225, 2015.

[3] J. C. Won, S. S. Kim, K. S. Ko, and B.-Y. Cha, "Current status of diabetic peripheral neuropathy in korea: Report of a hospitalbased study of type 2 diabetic patients in korea by the diabetic neuropathy study group of the korean diabetes association," Diabetes \& Metabolism, vol. 38, no. 1, pp. 25-31, 2014.

[4] N. Cho, J. Shaw, S. Karuranga et al., "IDF Diabetes Atlas: Global estimates of diabetes prevalence for 2017 and projections for 2045," Diabetes Research and Clinical Practice, vol. 138, pp. 271281, 2018.

[5] S. Tesfaye, A. J. Boulton, and P. J. Dyck, "Diabetic neuropathies: update on definitions, diagnostic criteria, estimation of severity, and treatment," Diabetes Care, vol. 33, no. 10, pp. 2285-2293, 2010.

[6] T. Didangelos, J. Doupis, and A. Veves, "Painful diabetic neuropathy: clinical aspects," Handbook of Clinical Neurology, vol. 126, pp. 53-61, 2014.

[7] J. W. Albers and R. Pop-Busui, "Diabetic neuropathy: mechanisms, emerging treatments, and subtypes," Current Neurology and Neuroscience Reports, vol. 14, p. 473, 2014.

[8] D. W. Zochodne, "Mechanisms of diabetic neuron damage: Molecular pathways," Handbook of Clinical Neurology, vol. 126, pp. 379-399, 2014.

[9] R. Wada and S. Yagihashi, "Role of advanced glycation end products and their receptors in development of diabetic neuropathy," Annals of the New York Academy of Sciences, vol. 1043, pp. 598-604, 2005.

[10] I. K. Lukic, P. M. Humpert, P. P. Nawroth, and A. Bierhaus, "The RAGE pathway: activation and perpetuation in the pathogenesis of diabetic neuropathy, Annals of the New York Academy of Sciences, vol. 1126, pp. 76-80, 2008.

[11] R. Ramasamy, S. F. Yan, and A. M. Schmidt, "Receptor for AGE (RAGE): signaling mechanisms in the pathogenesis of diabetes and its complications," Annals of the New York Academy of Sciences, vol. 1243, pp. 88-102, 2011.

[12] T. Purves, A. Middlemas, S. Agthong et al., "A role for mitogenactivated protein kinases in the etiology of diabetic neuropathy," The FASEB Journal, vol. 15, no. 13, pp. 2508-2514, 2001.

[13] R. Pop-Busui, A. J. M. Boulton, E. L. Feldman et al., "Diabetic neuropathy: A position statement by the American diabetes association," Diabetes Care, vol. 40, no. 1, pp. 136-154, 2017.

[14] N. Hotta, R. Kawamori, M. Fukuda, and Y. Shigeta, "Long-term clinical effects of epalrestat, an aldose reductase inhibitor, on progression of diabetic neuropathy and other microvascular complications: Multivariate epidemiological analysis based on patient background factors and severity of diabetic neuropathy," Diabetic Medicine, vol. 29, no. 12, pp. 1529-1533, 2012.

[15] D. Ziegler, P. A. Low, W. J. Litchy et al., "Efficacy and safety of antioxidant treatment with $\alpha$-lipoic acid over 4 years in diabetic polyneuropathy: the NATHAN 1 trial," Diabetes Care, vol. 34, no. 9, pp. 2054-2060, 2011.

[16] D. Ziegler, A. Ametov, A. Barinov et al., "Oral treatment with $\alpha$-lipoic acid improves symptomatic diabetic polyneuropathy," Diabetes Care, vol. 29, no. 11, pp. 2365-2370, 2006. 
[17] X. Yang, W. Yao, H. Liu, Y. Gao, R. Liu, and L. Xu, “Tangluoning, a traditional Chinese medicine, attenuates in vivo and in vitro diabetic peripheral neuropathy through modulation of PERK/Nrf2 pathway," Scientific Reports, vol. 7, no. 1, Article ID 1014, 2017.

[18] Y. Tong and H. Hou, "Effects of Huangqi Guizhi Wuwu Tang on diabetic peripheral neuropathy," The Journal of Alternative and Complementary Medicine, vol. 12, no. 6, pp. 506-509, 2006.

[19] A. Chen, H. Wang, J. Zhang et al., "BYHWD rescues axotomized neurons and promotes functional recovery after spinal cord injury in rats," Journal of Ethnopharmacology, vol. 117, no. 3, pp. 451-456, 2008.

[20] K.-J. Kim and U. Namgung, "Facilitating effects of Buyang Huanwu decoction on axonal regeneration after peripheral nerve transection," Journal of Ethnopharmacology, vol. 213, pp. 56-64, 2018.

[21] I. A. Chang, H. D. Lim, K. J. Kim, H. Shin, and U. Namgung, "Enhanced axonal regeneration of the injured sciatic nerve by administration of Buyang Huanwu decoction," Journal of Ethnopharmacology, vol. 194, pp. 626-634, 2016.

[22] T. Ji, "Clinical observation on treating diabetic peripheral neuropathy with Buyang Huanwu decoction," Clinical Journal of Chinese Medicine, vol. 6, pp. 74-75, 2014.

[23] J. Liu, "Efficacy observation on treating diabetic peripheral neuropathy with the Buyang Huanwu decoction," Clinical Journal of Chinese Medicine, vol. 7, pp. 173-174, 2015.

[24] H. J. Cui, W. R. Zhu, J. J. Zhou et al., "Clinical observation of compound xiongshao capsules in the treatment of diabetic peripheral neuropathy," China Pharmacy, vol. 29, pp. 223-228, 2018.

[25] D. Gao, Q. Li, Z. Liu et al., "Hypoglycemic effects and mechanisms of action of Cortex Lycii Radicis on alloxan-induced diabetic mice," Yakugaku Zasshi, vol. 127, no. 10, pp. 1715-1721, 2007.

[26] M. Ryu, E. H. Kim, M. Chun et al., "Astragali Radix elicits anti-inflammation via activation of MKP-1, concomitant with attenuation of p38 and Erk," Journal of Ethnopharmacology, vol. 115, no. 2, pp. 184-193, 2008.

[27] H. Z. Toklu, T. Tunali Akbay, A. Velioglu-Ogunc et al., "Silymarin, the Antioxidant Component of Silybum marianum, Prevents Sepsis-Induced Acute Lung and Brain Injury," Journal of Surgical Research, vol. 145, no. 2, pp. 214-222, 2008.

[28] S. Li and B. Zhang, "Traditional Chinese medicine network pharmacology: theory, methodology and application," Chinese Journal of Natural Medicines, vol. 11, pp. 110-120, 2013.

[29] J. Ru, P. Li, J. Wang et al., "TCMSP: a database of systems pharmacology for drug discovery from herbal medicines," Journal of Cheminformatics, vol. 6, no. 1, article 13, 2014.

[30] X. Deng, X. Xing, G. Sun et al., "Guanxin danshen formulation protects against myocardial ischemia reperfusion injuryinduced left ventricular remodeling by upregulating estrogen receptor beta," Frontiers in Pharmacology, vol. 8, p. 777, 2017.

[31] H. Ye, L. Ye, H. Kang et al., "HIT: Linking herbal active ingredients to targets," Nucleic Acids Research, vol. 39, no. 1, pp. D1055-D1059, 2011.

[32] L. Gao, X. Wang, Y. Niu et al., "Molecular targets of Chinese herbs: a clinical study of hepatoma based on network pharmacology," Scientific Reports, vol. 6, no. 1, Article ID 24944, 2016.

[33] L. Gao, K. Wang, Y. Zhou, J. Fang, X. Qin, and G. Du, "Uncovering the anticancer mechanism of Compound Kushen
Injection against HCC by integrating quantitative analysis, network analysis and experimental validation," Scientific Reports, vol. 8 , no. 624,2018 .

[34] J. Gong, C. Cai, X. Liu et al., "ChemMapper: a versatile web server for exploring pharmacology and chemical structure association based on molecular 3D similarity method," Bioinformatics, vol. 29, no. 14, pp. 1827-1829, 2013.

[35] L. Luo, L.-D. Ji, J.-J. Cai et al., "Microarray analysis of long noncoding rnas in female diabetic peripheral neuropathy patients," Cellular Physiology and Biochemistry, vol. 46, no. 3, pp. 12091217, 2018.

[36] T. Barrett, S. E. Wilhite, P. Ledoux et al., "NCBI GEO: archive for functional genomics data sets-update," Nucleic Acids Research, vol. 41, no. 1, pp. D991-D995, 2013.

[37] Y. Tian, B. Stamova, G. C. Jickling et al., "Effects of gender on gene expression in the blood of ischemic stroke patients," Journal of Cerebral Blood Flow \& Metabolism, vol. 32, no. 5, pp. 780-791, 2012.

[38] X. Chen, Z. L. Ji, and Y. Z. Chen, "TTD: therapeutic target database," Nucleic Acids Research, vol. 30, no. 1, pp. 412-415, 2002.

[39] D. S. Wishart, C. Knox, A. C. Guo et al., "DrugBank: a knowledgebase for drugs, drug actions and drug targets," Nucleic Acids Research, vol. 36, pp. D901-D906, 2008.

[40] M. Kanehisa, “The KEGG database," Novartis Foundation Symposia, vol. 247, pp. 91-103, 2002.

[41] J. Piñero, N. Queralt-Rosinach, À. Bravo et al., "DisGeNET: a discovery platform for the dynamical exploration of human diseases and their genes," Database, vol. 2015, Article ID bav028, 2015.

[42] A. Hamosh, A. F. Scott, J. S. Amberger, C. A. Bocchini, and V. A. McKusick, "Online Mendelian Inheritance in Man (OMIM), a knowledgebase of human genes and genetic disorders," Nucleic Acids Research, vol. 33, pp. D514-D517, 2005.

[43] M. Kuhn, C. von Mering, M. Campillos, L. J. Jensen, and P. Bork, "STITCH: interaction networks of chemicals and proteins," Nucleic Acids Research, vol. 36, no. 1, pp. D684-D688, 2008.

[44] M. E. Smoot, K. Ono, J. Ruscheinski, P. L. Wang, and T. Ideker, "Cytoscape 2.8: new features for data integration and network visualization," Bioinformatics, vol. 27, no. 3, pp. 431-432, 2011.

[45] H. de Jong, J. Geiselmann, C. Hernandez, and M. Page, “Genetic network analyzer: Qualitative simulation of genetic regulatory networks," Bioinformatics, vol. 19, no. 3, pp. 336-344, 2003.

[46] S. Li, Z. Q. Zhang, L. J. Wu, X. G. Zhang, Y. D. Li, and Y. Y. Wang, "Understanding ZHENG in traditional Chinese medicine in the context of neuro-endocrine-immune network," IET Systems Biology, vol. 1, no. 1, pp. 51-60, 2007.

[47] G. Bindea, B. Mlecnik, H. Hackl et al., "ClueGO: a Cytoscape plug-in to decipher functionally grouped gene ontology and pathway annotation networks," Bioinformatics, vol. 25, no. 8, pp. 1091-1093, 2009.

[48] N. P. Kumar, A. R. Annamalai, and R. S. Thakur, "Antinociceptive property of emblica officinalis gaertn (Amla) in high fat dietfed/low dose streptozotocin induced diabetic neuropathy in rats," Indian Journal of Experimental Biology (IJEB), vol. 47, no. 9, pp. 737-742, 2009.

[49] M. Anjaneyulu and K. Chopra, "Quercetin attenuates thermal hyperalgesia and cold allodynia in STZ-induced diabetic rats," Indian Journal of Experimental Biology (IJEB), vol. 42, no. 8, pp. 766-769, 2004. 
[50] P. E. B. Ferreira, C. R. P. Lopes, A. M. P. Alves et al., "Diabetic neuropathy: an evaluation of the use of quercetin in the cecum of rats," World Journal of Gastroenterology, vol. 19, no. 38, pp. 6416-6426, 2013.

[51] I. C. Chis, A. Clichici, A. L. Nagy, A. Oros, C. Catoi, and S. Clichici, "Quercetin in association with moderate exercise training attenuates injuries induced by experimental diabetes in sciatic nerves," Journal of Physiology and Pharmacology, vol. 68, pp. 877-886, 2017.

[52] L. Kishore, N. Kaur, and R. Singh, "Effect of Kaempferol isolated from seeds of Eruca sativa on changes of pain sensitivity in Streptozotocin-induced diabetic neuropathy," Inflammopharmacology, vol. 26, no. 4, pp. 993-1003, 2018.

[53] R. Stavniichuk, V. R. Drel, H. Shevalye et al., "Baicalein alleviates diabetic peripheral neuropathy through inhibition of oxidativenitrosative stress and p38 MAPK activation," Experimental Neurology, vol. 230, no. 1, pp. 106-113, 2011.

[54] X. Wang, L. I. Cai-Na, and Z. F. J. C. J. o. C. P. Shen, "Advances on the protective mechanism study of baicalein on diabetic peripheral neuropathy," 2018.

[55] Y. Shi, X.-C. Liang, H. Zhang, Q.-L. Wu, L. Qu, and Q. Sun, "Quercetin protects rat dorsal root ganglion neurons against high glucose-induced injury in vitro through Nrf-2/HO-1 activation and NF- $\kappa$ B inhibition," Acta Pharmacologica Sinica, vol. 34, no. 9, pp. 1140-1148, 2013

[56] L. Shi, X. Yu, H. Yang, and X. Wu, "Advanced glycation end products induce human corneal epithelial cells apoptosis through generation of reactive oxygen species and activation of JNK and p38 MAPK pathways," PLoS ONE, vol. 8, no. 6, Article ID e66781, 2013.

[57] S. J. Harper and P. LoGrasso, "Signalling for survival and death in neurones: the role of stress-activated kinases, JNK and p38," Cell Signal, vol. 13, pp. 299-310, 2001.

[58] K. Takeda and H. Ichiijo, "Neuronal p38 MAPK signalling: An emerging regulator of cell fate and function in the nervous system," Genes to Cells, vol. 7, no. 11, pp. 1099-1111, 2002.

[59] J. C. Lee, S. Kumar, D. E. Griswold, D. C. Underwood, B. J. Votta, and J. L. Adams, "Inhibition of p38 MAP kinase as a therapeutic strategy," International Journal of immunopharmacology, vol. 47, no. 2-3, pp. 185-201, 2000.

[60] G.-H. Jeohn, C. L. Cooper, B. Wilson et al., "p38 MAP kinase is involved in lipopolysaccharide-induced dopaminergic neuronal cell death in rat mesencephalic neuron-glia cultures," Annals of the New York Academy of Sciences, vol. 962, pp. 332346, 2002.

[61] R. Stavniichuk, H. Shevalye, H. Hirooka, J. L. Nadler, and I. G. Obrosova, "Interplay of sorbitol pathway of glucose metabolism, 12/15-lipoxygenase, and mitogen-activated protein kinases in the pathogenesis of diabetic peripheral neuropathy," Biochemical Pharmacology, vol. 83, no. 7, pp. 932-940, 2012.

[62] H. Cheng, J. Dauch, J. Hayes, Y. Hong, and E. Feldman, "P38 mediates mechanical allodynia in a mouse model of type 2 diabetes," Molecular Pain, vol. 6, no. 28, 2010.

[63] V. R. Drel, P. Pacher, R. Stavniichuk et al., "Poly(ADP-ribose) polymerase inhibition counteracts renal hypertrophy and multiple manifestations of peripheral neuropathy in diabetic Akita mice," International Journal of Molecular Medicine, vol. 28, no. 4, pp. 629-635, 2011.
[64] L. Daulhac, C. Mallet, C. Courteix et al., "Diabetes-induced mechanical hyperalgesia involves spinal mitogen-activated protein kinase activation in neurons and microglia via N-methylD-aspartate- dependent mechanisms," Molecular Pharmacology, vol. 70, no. 4, pp. 1246-1254, 2006.

[65] L. Daulhac, V. Maffre, C. Mallet et al., "Phosphorylation of spinal N-methyl-d-aspartate receptor NR1 subunits by extracellular signal-regulated kinase in dorsal horn neurons and microglia contributes to diabetes-induced painful neuropathy," European Journal of Pain, vol. 15, no. 2, pp. 169-e12, 2011.

[66] S. A. Price, N. J. Gardiner, B. Duran-Jimenez, L. A. H. Zeef, I. G. Obrosova, and D. R. Tomlinson, "Thioredoxin interacting protein is increased in sensory neurons in experimental diabetes," Brain Research, vol. 1116, no. 1, pp. 206-214, 2006.

[67] S. A. Price, S. Agthong, A. B. Middlemas, and D. R. Tomlinson, "Mitogen-activated protein kinase p38 mediates reduced nerve conduction in experimental diabetic neuropathy: interactions with aldose reductase," Diabetes, vol. 53, no. 7, pp. 1851-1856, 2004.

[68] H.-T. Liu, Y.-G. Du, J.-L. He et al., “Tetramethylpyrazine inhibits production of nitric oxide and inducible nitric oxide synthase in lipopolysaccharide-induced N9 microglial cells through blockade of MAPK and PI3K/Akt signaling pathways, and suppression of intracellular reactive oxygen species," Journal of Ethnopharmacology, vol. 129, no. 3, pp. 335-343, 2010.

[69] Y. S. Kanwar, L. Sun, P. Xie, F. Y. Liu, and S. Chen, "A glimpse of various pathogenetic mechanisms of diabetic nephropathy," Annual Review of Pathology, vol. 6, pp. 395-423, 2011. 


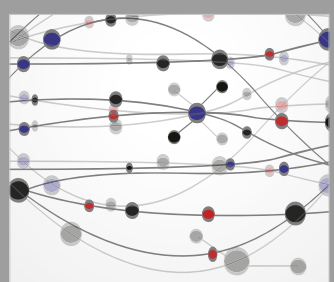

The Scientific World Journal
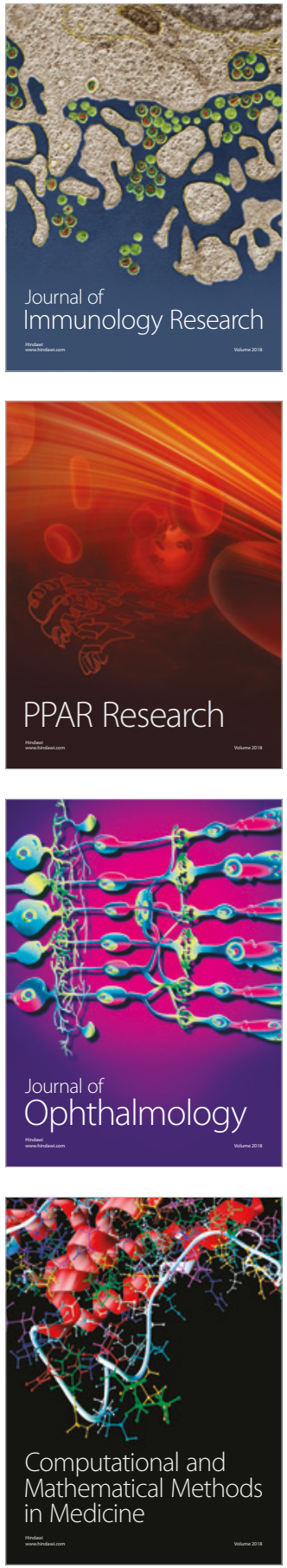

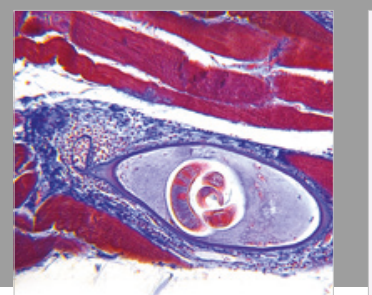

Gastroenterology Research and Practice

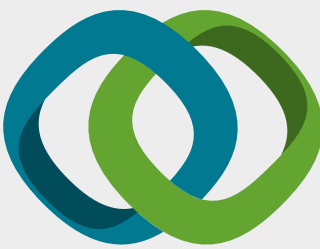

\section{Hindawi}

Submit your manuscripts at

www.hindawi.com
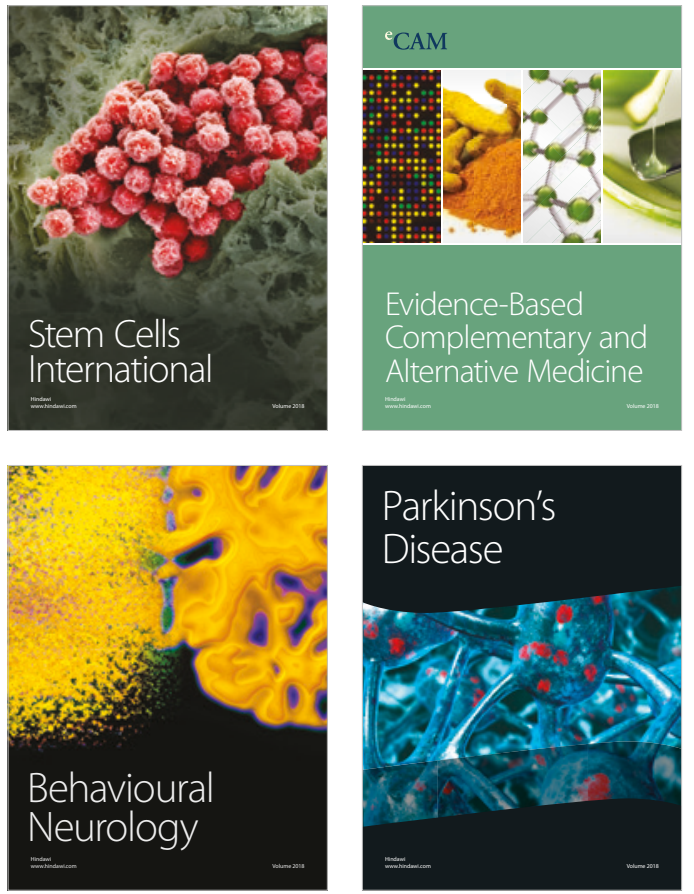

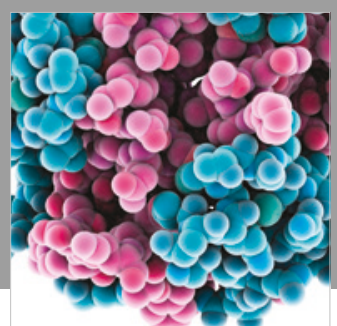

ournal of

Diabetes Research

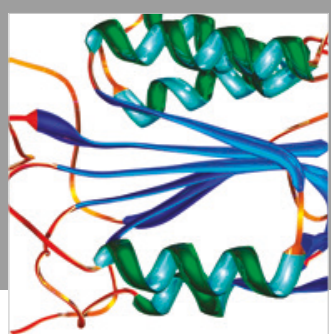

Disease Markers
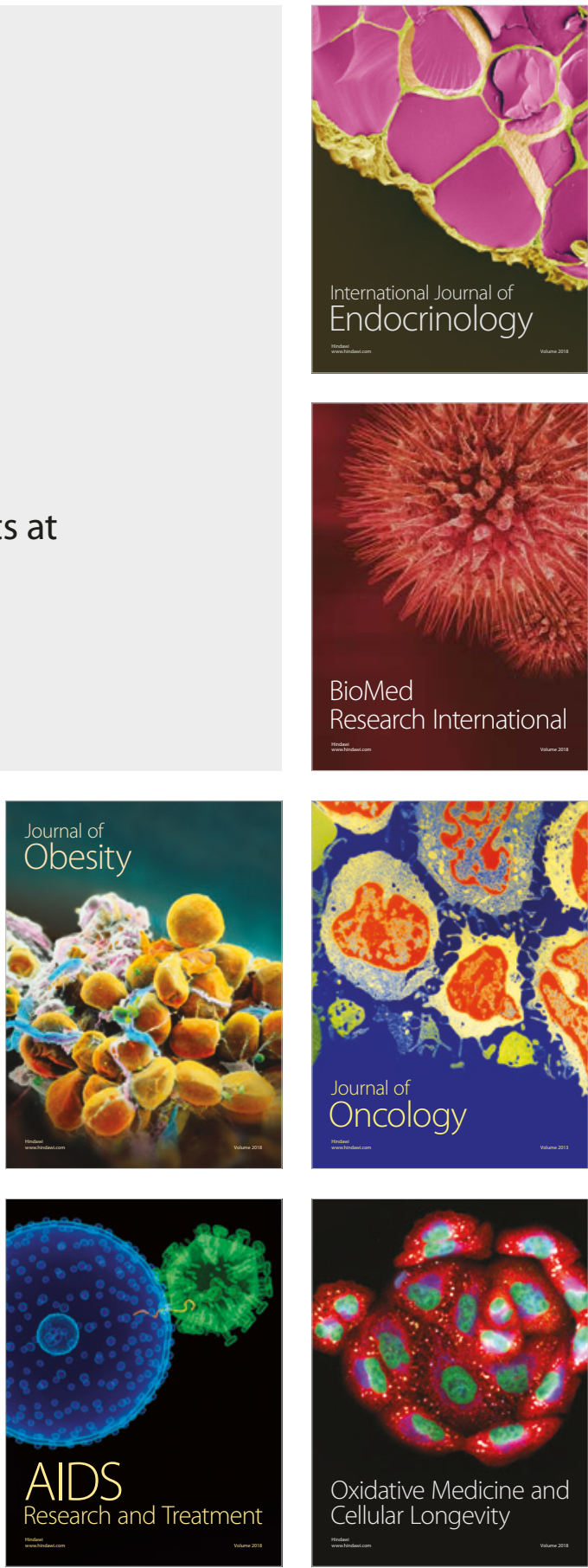